\title{
Inter-Proximal Space Management Using Indirect Ceramic Veneers
}

\author{
Authors \\ Dr Maha Alwaqeet, Dr Lamees Albedaiwi, Dr Ghaida Alduhayan, Dr Alaa Alotaibi, \\ Dr Abdullah Bin Dawas
}

\begin{abstract}
Teeth spacing defined as a dental anomaly characterized by interdental spaces and lack of contact points between the teeth, the etiology of spacing in dentition may be hereditary, acquired or functional. Using the appropriate technique and material for effective treatment are based on time, physical, psychological and economical limitations. Possible treatment options include orthodontics, restorative dentistry, surgery and various combinations of the above. All-ceramic systems for dental restoration have been extensively used over recent years due to substantial developments meeting dental requirements, particularly in terms of their mechanical properties and the opaque, presently accessible appearance of all-ceramic materials. In this case report inter-proximal space management was done using proximal ceramic veneers without any preparation. At one year recall session the veneers were checked both clinically and radiographically, the patient and the team were satisfied with the esthetic and functional outcomes of the definitive restorations.

Keywords: Teeth Spacing, Spaced Dentition, Proximal Veneers, Inter-proximal Contact Closure.
\end{abstract}

\section{INTRODUCTION}

According to the epidemiological study of Steigman and Weissberg (1985), 21.4\% of the general population presented spacing in both arches, whereas $50 \%$ of people with spacing had spaces in both arches. ${ }^{[1]}$

The etiology of spacing may be hereditary, acquired or functional. Hereditary causes include tooth size - arch size discrepancies, congenitally missing teeth, macroglossia, supernumerary teeth, small teeth and hypertrophic upper lip frenum. Functional causes include deleterious oral habits, whereas acquired causes include pathlogic conditions increasing tongue size, missing teeth, delayed eruption of permanent teeth and periodontal disease. ${ }^{[1]}$
Once the etiology is clear, a treatment can be determined. Treatment options range from a no treatment or esthetic restoration, an Orthodontic space closure, or a retention or further space opening for prosthetic rehabilitation. ${ }^{[1]}$ The closing of diastemas in the posterior area was described by Vest as early as 1951. ${ }^{[2]}$ As it is important to restore proximal contact because it is both supportive and protective in nature. ${ }^{[3]}$

All-ceramic systems for dental restoration have been extensively used over recent years due to substantial developments meeting dental requirements, particularly in terms of their mechanical properties and the opaque, presently accessible appearance of all-ceramic materials. Lithium disilicate glass ceramic is one such all-ceramic system, currently used in the fabrication of single 


\section{JMSCR Vol||05||Issue||01||Page 15845-15849||January}

and multiunit dental restorations mainly for dental crowns, bridges, and veneers because of its color being similar to natural teeth and its excellent mechanical properties. ${ }^{[4]}$

Dental ceramic materials exhibits many desirable material properties, including biocompatibility, esthetics, diminished plaque accumulation, low thermal conductivity, abrasion resistance, and color stability. ${ }^{[5]}$ Thus becoming an alternative to traditional restorative materials. ${ }^{[6]}$

\section{CASE REPORT}

A 52-year-old female patient reported to the dental department of King Saud University Medical City with a chief complaint of space in her lower left posterior teeth. No systemic diseases or drug allergies were noted.

Intraoral examination revealed an approximately 3 $\mathrm{mm}$ spacing between the mandibular left second premolar and second molar. [Figure1] No dental caries were found upon clinical and radiographic examination. The patient demonstrated good periodontal health with reduced periodontium.

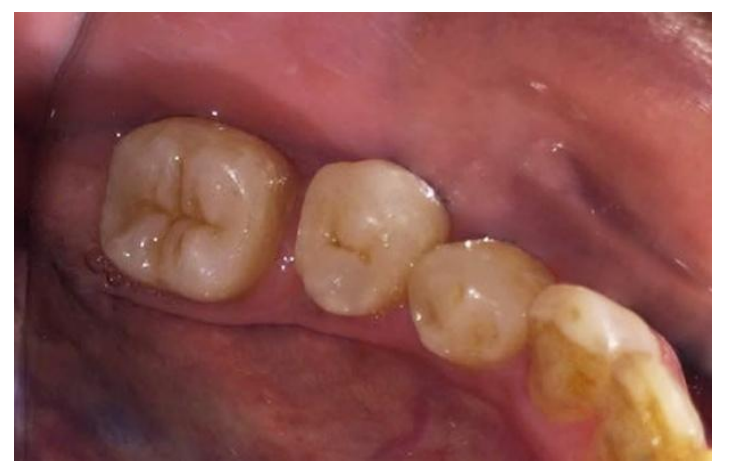

[Figure1]: Pre-Operative Intra-oral picture showing $3 \mathrm{~mm}$ space between mandibular left-second premolar and molar.

After discussing the patient with the different treatment options, the patient's desire for immediate results and due to financial constraints the treatment plan included closing the space with proximal ceramic veneers. A study model was fabricated to assess the proximal undercuts by using dental surveyor (NEY® Surveyor, Dentsply, UK). [Figure2]

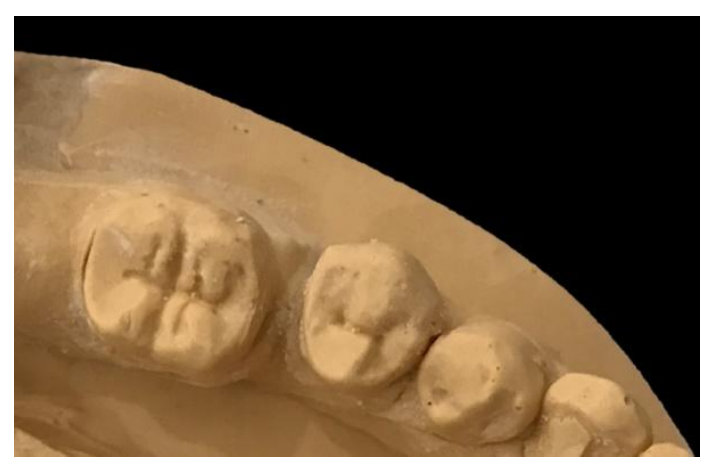

[Figure2] Study Cast.

Shade selection was done using shade guide (A-D SHADE GUIDE, Ivoclar-Vivadent ${ }^{\circledR, S c h a a n, ~}$ Liechtenstein) and shade A2 was selected. Final impression was made using light body

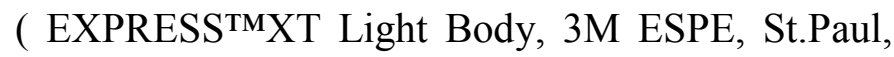
Mn, USA ) and regular body Vinyl Polysiloxane impression ( EXPRESS ${ }^{\mathrm{TM}}$ XT Penta ${ }^{\mathrm{TM}} \mathrm{H}, 3 \mathrm{M}$ ESPE, St.Paul, Mn, USA ) and master cast was fabricated and pressed ceramic veneers ( IPS E.MAX, IvoclarVivadent ${ }^{\circledR}$, Schaan, Liechtenstein) were fabricated for mandibular left second premolar and second molar. [Figure3]

The definitive restorations were checked in the patient's mouth and the proximal and buccal surfaces of mandibular left second premolar and second molar were etched with $32 \%$ phosphoric acid (SCOTCHBOND ${ }^{\mathrm{TM}}$ Universal Etchant, 3M ESPE, St.Paul, Mn, USA) for 15 seconds, followed by washing and drying with absorbent cotton pellets. Next, the bonding agent (SINGLE BOND UNIVERSAL, 3M ESPE, St.Paul, Mn, USA ). Was applied without curing.

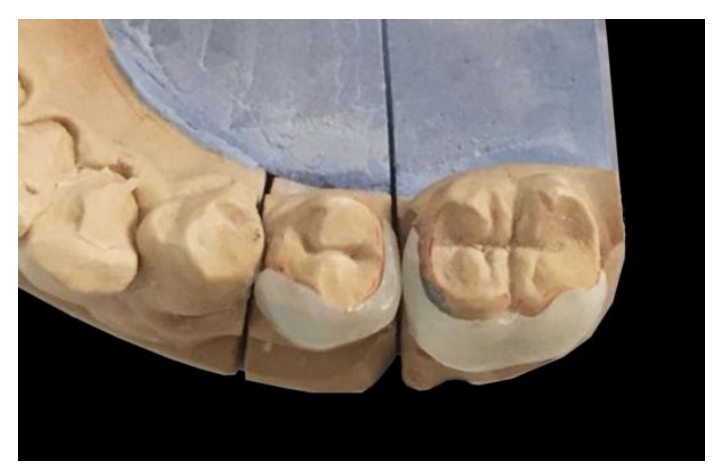

[Figure3] Ceramic Veneers fabricated on master cast. 
The internal surfaces of the veneers were etched using 9.5\% hydrofluoric acid gel (BISCO Dental Products, Schaumburg, IL, USA) for 20 seconds followed by application of Silane agent (ULTRADENT®, South Jordan, UT, USA) for 60 seconds.

The resin cement (RELYX $^{\text {TMUltimate }}$ ClickerTM 3M ESPE, St.Paul, Mn, USA ) was applied into the internal part of the veneers which were placed onto the tooth surface of mandibular left second premolar and cured for 2 seconds then the cement excesses were removed and final curing for 40 seconds on the veneer was done. Same procedures were carried out on the mandibular left second molar.

The occlusion was checked on both centric and eccentric relations using $40 \mu$ articulating paper. The contact was checked using dental floss and the finishing procedure was carried out using composite finishing burs followed by ceramic polishing bur. [Figure4] The patient was motivated to maintain good oral hygiene and informed for a recall session every 6 months.
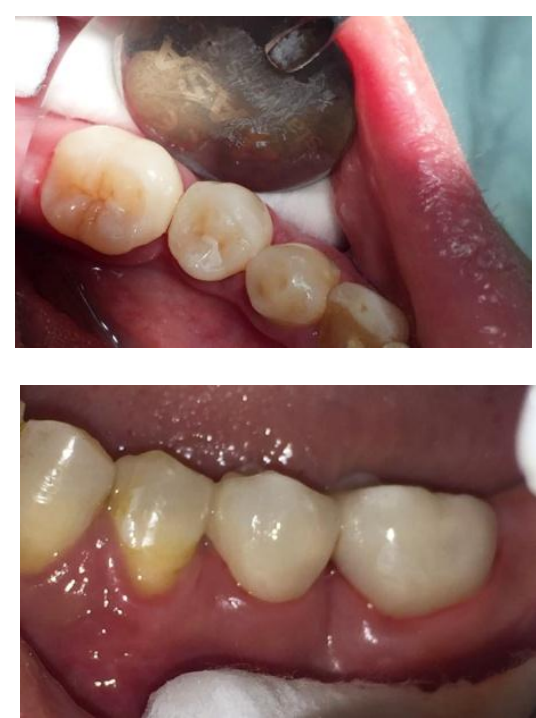

[Figure4]: Post-Operative intra-oral picture showing ceramic Veneers after cementation.

At one year recall session the veneers were checked both clinically and radiographically, the patient and the team were satisfied with the esthetic and functional outcomes of the definitive restorations. [Figure5]

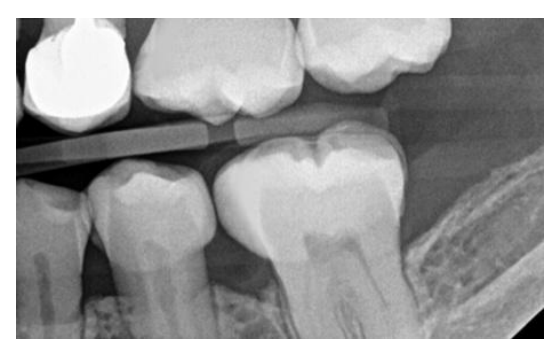

[Figure5] Post-Operative one year recall radiograph.

\section{DISCUSSION}

In dentistry it is quit usual to adapt a material from engineers and adapt it to clinical conditions. Dental ceramic is a good example of such material. In Dental science, ceramics are defined as nonmetallic, inorganic structures primarily containing compounds of oxygen with one or more metallic or semi-metallic elements. Such as sodium, potassium, calcium, magnesium, aluminum, silicon, phosphorus, zirconium and titanium. These materials exhibit many desirable properties, including biocompatibility, aesthetics, durability and easily customized. In addition ceramics have excellent intraoral wear resistance and stability. ${ }^{[7]}$

Nevertheless, there are some disadvantages that ceramics possess making case selection extremely crucial. Dental ceramics are higher in price than regular composites. But in our case it was done at a government hospital with no treatment charge. Secondly, unlike composite, ceramics are not gentle on opposing dentition. However, our veneer was out of occlusion hence use of ceramics would have no effect on opposing. ${ }^{[8]}$

All-ceramic systems can offer a higher esthetic result for a wide scope of patients, because a wide range of translucency-opacity can be accomplished with the commercially accessible ceramic systems. Moreover, ceramics relate as much to soft tissue health as to esthetics. Lower amounts of plaque and adherence molecules are retrieved from ceramic surfaces than from amalgam or gold alloys, and intra-oral plaque of a qualitatively healthier composition can form on ceramic surfaces. Furthermore, it is usually acceptable to leave the margin of all-ceramic prostheses at the gingival margin or supragingival, and the advantage of more foreseeable and less traumatic impression making. ${ }^{[9]}$ 
Porcelain veneer restorations require experience and skills to have a tight proximal contact since failure to have a tight proximal contact is one of the challenges that the clinicians face. B.A.C.Loomans reported that after 6 months period the tightness in posterior proximal contacts which are stronger before composite resin treatment tends to diminish.[10]

Ceramics are especially suitable for veneer restorations. Porcelain veneers fabricated from different types of ceramics, as reported from eleven studies of generally 3 to 5 years duration have failure rates (loss of retention or fracture) of 5\%. ${ }^{[9]}$

In our case, try-in was difficult to manage since its proximal veneers. Moreover, Restoring mandibular teeth is much more difficult than on maxillary teeth, This is due isolation. However, isolation was managed by using cotton roll.

The ceramics used for posterior diastema closure must have the ability to mimic the natural tooth in translucency, color, and strength. In the present case we used (IPS E.max, Ivoclar-Vivadent ${ }^{\circledR}$, Schaan, Liechtenstein) which is well suited for this purpose. [7]

Ella A. NAUMOVA et al. (2016) reported that RelyX Ultimate Clicker cement showed less adhesive failure pattern compared to Variolink II, and higher median shear bond strength to enamel and dentin. ${ }^{[11]}$

Recent studies have concluded that Ceramics are widely used in dentistry due to their ability to mimic the optical characteristics of enamel and dentin and their biocompatibility and chemical durability. [9] Similarly, Porcelain is stronger and stains less than composite; however, it costs more. Porcelain is difficult to customize to a demanding patient. However, the results of porcelain are excellent when using an excellent laboratory. ${ }^{[12]}$

\section{CONCLUSION}

Considering the final result of this case report, it can be concluded that ceramic veneers provided satisfactory esthetic and functional outcome to close the space between the teeth.

\section{REFERENCES}

1. Gkantidis, N., Teeth spacing: etiology and treatment. Hellenic Orthodontic Review 2007. Volume 10 (Issue 2).

2. Wolff, D., et al., Proximal contact tightness between direct-composite additions in the posterior dentition: an in vitro investigation. Oper Dent, 2012.37 (3) : p. 272-80.

3. DA., T., Restoring the interproximal zone using the proximal adaptation technique-Part 2.CompendContinEduc Dent. 2005;26:152.

4. Monmaturapoj, N., P. Lawita, and W. Thepsuwan, Characterisation and Properties of Lithium Disilicate Glass Ceramics in the SiO2-Li2O-K2OA12O3System for Dental Applications. Advances in Materials Science and Engineering, 2013.2013: p. 1-11.

5. Tinschert J, N.G., Mautsch W, Augthun M, Spiekermann H., Fracture resistance of lithium disilicate-, alumina-, and zirconia-based three-unit fixed partial dentures: a laboratory study.Int J Prosthodont. 2001 May-Jun;14(3):231-8.

6. Blatz, M.B., Long-term clinical success of all-ceramic posterior restorations. Quintessence International, 2002. Vol. 33 Issue 6).

7. Raghavan, R.N., Ceramics in Dentistry, Sintering of Ceramics - New Emerging Techniques, Dr. Arunachalam Lakshmanan (Ed.), ISBN: 978-953-51-0017-1, InTech.

8. Goyal, A., V. Nikhil, and R. Singh, Diastema Closure in Anterior Teeth Using a Posterior Matrix. Case Rep Dent, 2016. 2016: p. 2538526.

9. Kelly, J.R., Dental ceramics: current thinking and trends. Dent Clin North Am, 2004. 48(2): p. viii, 513-30.

10. Loomans, B.A., et al., The long-term effect of a composite resin restoration on 
proximal contact tightness. J Dent, 2007. 35(2): p. 104-8.

11. Naumova, E.A., et al., Adhesion of different resin cements to enamel and dentin. Dent Mater J, 2016. 35(3): p. 345-52.

12. Paul R. Chalifoux, D., Diastema Closure. inside dentistry, 2010.Volume 6(Issue 8). 